\title{
CARDIAC FAILURE AND PPHN DUE TO EXTRA CARDIAC CAUSE IN NEWBORN (VEIN OF GALEN MALFORMATION)
}

\author{
Dinakara Prithviraj ${ }^{1}$, Suresh ${ }^{2}$, Anna Mariam Paul ${ }^{3}$
}

\section{HOW TO CITE THIS ARTICLE:}

Dinakara Prithviraj, Suresh A, Anna Mariam Paul. "Cardiac Failure and PPHN due to Extra Cardiac Cause in Newborn (Vein of Galen Malformation)". Journal of Evolution of Medical and Dental Sciences 2014; Vol. 3, Issue 15, April 14; Page: 3945-3950, DOI: 10.14260/jemds/2014/2381

ABSTRACT: Vein of Galen aneurysmal malformations (VGAM) are rare intracranial vascular anomalies typically found in children. The anatomic landmark of a VGAM is the presence of multiple arteriovenous shunts draining into a dilated median prosencephalic vein, an embryonic vessel normally absent at the adult stage. This article reviews the developmental anatomy, the clinical presentation, and the current management of VGAM.

KEYWORDS: Aneurysmal malformations of the vein of Galen, Trans catheter embolization (TCE), cardiac failure.

INTRODUCTION: The vein of Galen is located under the cerebral hemispheres and drains the anterior and central regions of the brain into the sinuses of the posterior cerebral fossa. The vein of Galen aneurysmal malformation is a choroidal type of arteriovenous malformation involving the vein of Galen forerunner and is distinct from an arteriovenous malformation with venous drainage into a dilated, but already formed, vein of Galen. ${ }^{1}$

Aneurysmal malformations of the vein of Galen (VGAM) typically result in high-output congestive heart failure or may present with developmental delay, hydrocephalus, and seizures.

MATERIALS AND METHODS: Here we present a case, a term male newborn appropriate for gestational age referred to us for respiratory distress, grunting and increased precordial activity and cardiac murmur. On extensive evaluation was found to have in MRI brain scan with Venogram revealed a huge vein of Galen aneurysm /malformation with multiple feeders (Choroidal in type).

A brief review of literature is discussed.

CASE PRESENTATION: A six hours old male child born to a primigravida mother out of nonconsanguineous marriage. Baby cried immediately after birth and APGAR score was good.

Birth weight 3kgs.Mild respiratory distress noted at birth and was admitted in the NICU and given supplemental Oxygen and IV fluids. Gradually respiratory distress increased, with evidence of grunting, increased precordial pulsations, $\mathrm{SPO}_{2}$ fluctuations and cardiac murmur . Chest X-ray revealed Cardiomegaly. (Fig: 2) Referred to us for further management.

On admission baby was noted to have grunting, tachypnea, sternal, subcostal and intercostal retractions, mild precordial and epigastric pulsations (Fig: 1 ).

HR: 188/min., RR 110/min., SPO 2 80-85\%fluctuating, BP: 40/30/38mmHg, CFT: 4 secs, all peripheral pulses felt but feeble, color appeared pale. With nasal prong Oxygen, $\mathrm{SPO}_{2}$ improved to $92 \%$.

On systemic examination: CVS: S1 S2 heard, loud P2 present and systolic murmur of grade $4 / 6$ was present. Respiratory system: respiratory distress present and bilateral air entry was equal. 
Abdominal examination: Right hypochondriac fullness present, liver enlarged $4 \mathrm{~cm}$ below the RCM.

CNS examination; normal AF and irritability present, tone and reflexes were normal.

Chest X-ray revealed cardiomegaly. Echocardiography revealed severe pulmonary artery hypertension, tricuspid regurgitation, enlargement of right atrium and right ventricle, dilatation of great vessels and no structural cardiac abnormalities.

Blood was taken for all septic work-up. At this present clinical scenario with normal hematological findings (Hb:17.9g\%, TC:17, 000/mm3, DC:P-78\%, L-19\%, E-3\%, platelets: $260000 / \mathrm{mm} 3$ ), we started nasal prong Oxygen. Digoxin, furosemide, fluid restriction and inotrope (Dopamine).

Gradually over six hours baby started passing urine, SPO2 increased to $96 \%$ and heart rate came down to $170 / \mathrm{min}$. On second day, the calculated urine output was $<1 \mathrm{ml} / \mathrm{kg} / \mathrm{hr}$. and baby was also noted to have mild hematuria, jaundice (serum bilirubin Total:10mg/dl and direct: $1 \mathrm{mg} / \mathrm{dl}$ ), liver $5 \mathrm{~cm}$ below the RCM, mild abdominal distension, HR:160-170/min, RR:80-90/min with increase in drawing, CFT:>4secs, BP:45/35/42mm Hg SPO2 89-90\% fluctuating, increase in precordial pulsations and color appeared pale.

On second opinion for cardiac status, the reports were same (ECHO). (Fig: 4\&5) Baby was kept on CPAP $5 \mathrm{~cm}$ with $80 \%$ Oxygenation and $\mathrm{SPO}_{2}$ was stabilized to $>95 \%$.Continued the same treatment along with phototherapy for 2 days.On day 4 , the urine output was $0.9 \mathrm{ml} / \mathrm{kg} / \mathrm{hr}$., urea: $68 \mathrm{mg} / \mathrm{dl}$, creatinine: $1.8 \mathrm{mg} / \mathrm{dl}$, serum bilirubin: $14 \mathrm{mg} / \mathrm{dl}$.

Baby's irritability increased, perspiration noted, in drawing increased, murmur was of same intensity, liver: $5.5 \mathrm{~cm}$ below the right costal margin, all peripheral pulses were bounding, neck pulsations were prominent and mild bleeding from the nasogastric tube noted.

Repeat chest X-ray revealed cardiomegaly with mild increase in pulmonary markings. With all these features we suspected extra cardiac problem for the cardiac failure, with high index of suspicion of $\mathrm{A}-\mathrm{V}$ malformation/ aneurysm the cranium examination revealed Bruit.

Bedside cranial ultrasound was done which revealed huge vein of Galen aneurysm with shifting of the right lateral ventricles without hydrocephalus. (Fig: 7)

At the same time, ultrasound abdomen revealed hepatosplenomegaly, ascites and gall bladder wall congestion which were suggestive of congestive heart failure. (Fig: 6)

For further confirmation MRI brain scan with Venogram was carried out which revealed huge vein of Galen aneurysm / malformation with multiple feeders (Choroidal in type).

(Fig: 8 \& 9)Neurosurgeon's opinion was later taken. After collective decision about baby's poor prognosis and financial burden for parents we decided to withdraw the treatment.

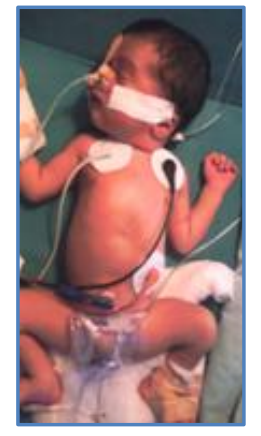

Fig. 1: PRECORDIAL BULGE \& RIGHT HYPOCHONDRIAC FULLNESS 


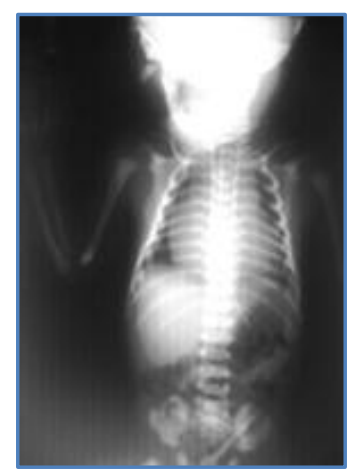

Fig. 2: INFANTOGRAM REVEALING CARDIOMEGALY

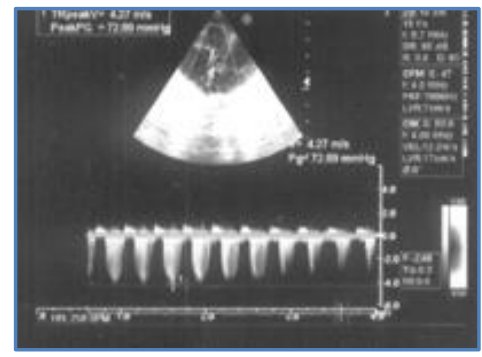

Fig. 4: 2D ECHO TRICUSPID REGURGITATION

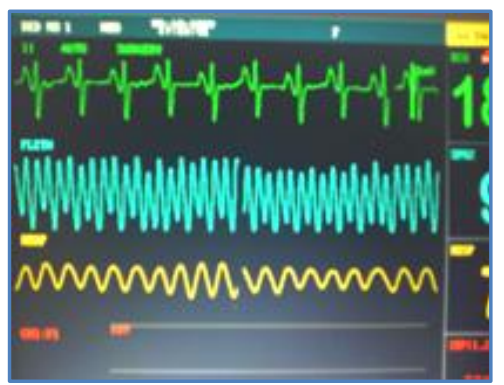

Fig. 3: SPO2 MONITOR REVEALING TALL \& WIDE P WAVES

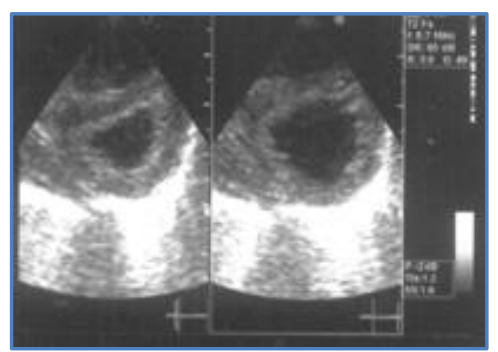

Fig. 5: 2D ECHOD SHAPED LEFT VENTRICLE

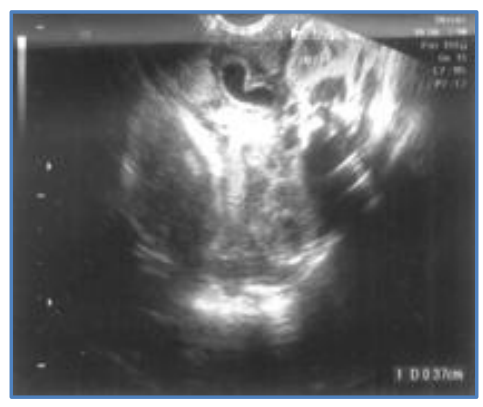

Fig. 6: USG ABDOMEN EDEMATOUS GALL BLADDER
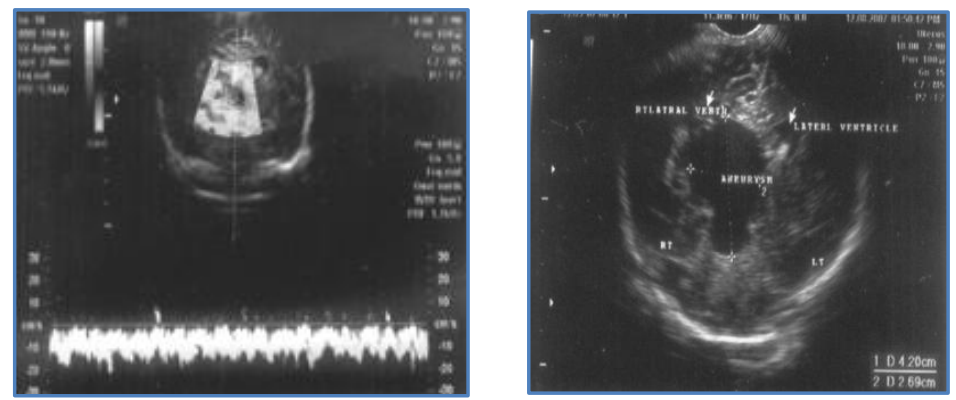

Fig. 7: DOPPLER STUDY OF VEIN OF GALEN \& MARKINGS IN U/S BRAIN 


\section{CASE REPORT}

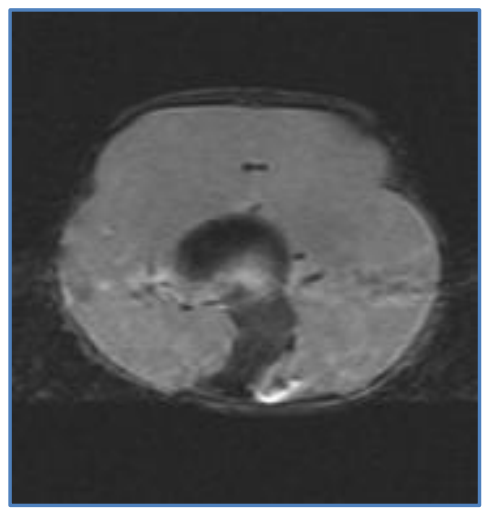

\section{Fig. 8: M R I PICTURE CONFIRMING} VEIN OF GALEN MALFORMATION
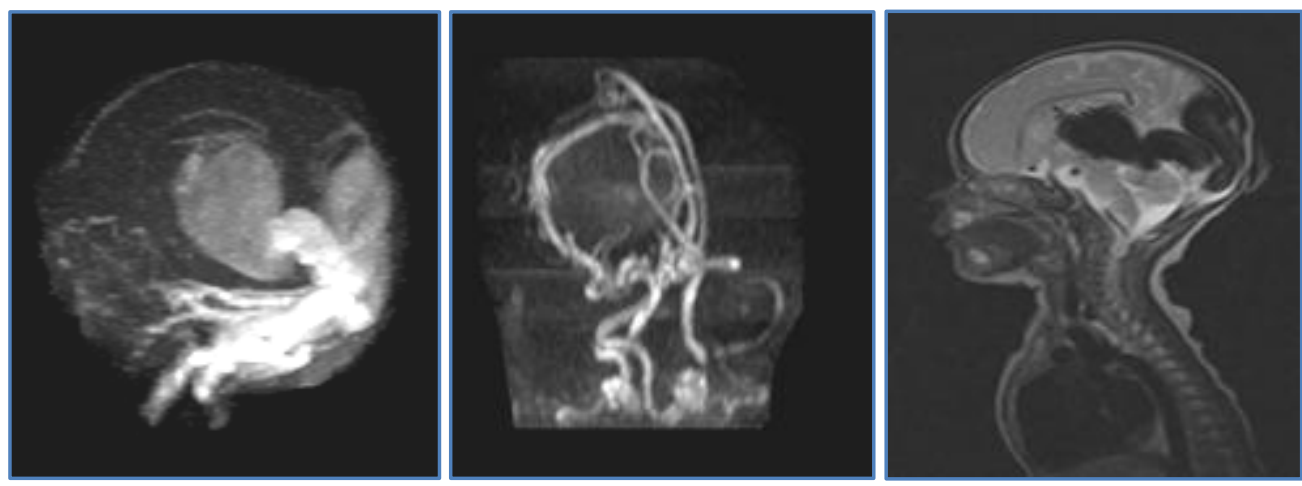

\section{Fig. 9: MRI VENOGRAM SHOWING VEIN OF GALEN} MALFORMATION AND FEEDING ARTERIES

DISCUSSION: Vein of Galen malformation is not a true aneurysm. It is only an Arterio- venous fistula (AVF) between deep choroidal arteries and the median prosencephalic vein (MPV) of Markowski. Because of this fistula, vein of Galen is not able to form an aneurysm. It is only a pooling site (Pouch). ${ }^{2}$ Abnormal connection of choroidal arteries to median prosencephalic vein (MPV) occurs at six to eleven weeks of gestation. Flow through this fistula prevents normal regression of MPV, so multiple abnormal connections will develop. It is sporadic in occurrence and there is no increased risk to siblings. Male: female ratio is 2:1. It is most commonly diagnosed in the newborn period and rarely after three years.

If diagnosed in older children or adults, it is usually compensated. It comprises less than $1 \%$ of cerebral vascular malformations at any age, but in the pediatric vascular malformation group it might account up to $30 \%$. It is the most common extra cardiac cause of high output cardiac failure in newborns. Sometimes it may be associated with ASD (may exacerbate CHF) and aortic coarctation. ${ }^{3}$

Probable causes include (assumption): a) venous occlusion/ stenosis leading to increased flow and pressure. b) Cerebral ischemia/atrophy leading to arterial steal and /or chronic venous hypertension. c) Hydrocephalus due to decreased CSF resorption or cerebral aqueductal obstruction. ${ }^{4}$

Sometimes it might cause malformation of adjacent brain structures like Pineal gland and third ventricle. Microscopically wall of the venous pouch is thickened. 


\section{CASE REPORT}

The classification (based on angio-architecture):

1. Choroidal type has multiple feeders from pericallosal, choroidal and thalamo perforating arteries.

2. Mural type: has single or few feeders from collicular or posterior choroidal arteries. 5

Clinically most common signs/symptoms: a) In Neonate: high-output cardiac failure and cranial bruit. b) In infants and older children: Macrocrania, prominant superficial cranial veins, developmental delay, failure to thrive, hydrocephalus, seizures, headache and hepatic failure. ${ }^{6}$

Imaging Findings: Ultrasound: a) Prenatal studies can identify malformation in second and third trimester. Cardiac dilatation and hydrops foetalis carries poor prognosis. b) Postnatally it is useful for early bedside diagnosis, provided there is large midline varix located in quadrigerminal plate cisterna. Size can vary upto several centimeters. Morphologically it might look spherical / tubular varix.

Echocardiography: Shows dilatation of right heart, SVC and ascending aorta, severe pulmonary artery hypertension and PDA with significant R-L shunt (poor prognosis).

MRI Findings: Fetal MR can identify malformation along with absence of brain or other end organ injury (significant antenatal end organ injury is a contraindication to aggressive treatment). MRA (magnetic resonance angiography) study is the key for pre-treatment assessment of feeders to malformation (MRA is unaffected by coils or acrylic embolic material). MRV (magnetic resonance venogram) is essential in initial and follow-up evaluation (presents and degree of venous stenosis can have major influence on prognosis). Varix contents are hypointense and homogenous (T1W signal), sharp delineation of malformation and prominent flow voids from feeding arteries around varix (T2W signal).

Prognosis is related to volume of shunt and timing/ success of treatment. High volume shunts requiring treatment in newborn period have worst prognosis. Delay in treatment until four to six months is associated with better outcome. Without treatment, progression of CHF or brain damage results in death. Up to $60 \%$ will be neurologically normal after treatment.

Treatment: Intractable $\mathrm{CHF}$, multi organ failure and brain damage at presentation are contraindication to treatment.

Medical therapy for CHF until four to six months (failure of therapy warrants earlier neurointervention).

Trans catheter embolization (TCE) at four to six months is the best available treatment at present. Permanent occlusion of fistula point from arterial side is done. It may require several stages of embolization (upto five to seven stages). Filling of venous pouch is less effective Frequent neurological and MRI assessment after TCE is needed. If deterioration is noted, further embolisations are needed.

Treatment for hydrocephalus is controversial and is reserved for refractory hydrocephalus after all TCEs performed. Because shunt placement is associated with exacerbation of venous ischemia and there is risk of hemorrhage from engorged sub ependymal veins. Over all these procedures are cost effective (3-5 lakhs). ${ }^{7}$ 
CONCLUSION: In our case, baby had high volume shunt and progressive multi organ failure, poor prognostic indicators were present, and also In view of financial aspects parents were not willing to continue the treatment and baby was discharged at request.

\section{REFERENCES:}

1. Rennie JM, Roberton NRC. CNS malformations, Textbook of Neonatology $3^{\text {rd }}$ edn; 1309.

2. Kliegman RM, Behrman RE, Jenson HB. Diseases of the Peripheral vascular System. Nelson Textbook of Pediatrics $18^{\text {th }}$ edn; 1988-1995

3. Abernethy LJ. Classification and imaging of vascular malformations in children. Eur Radiol 2003;13:2483-2497

4. Friedman DM, Verma R, Madrid M et al. Recent improvement in outcome using transcatheter embolization techniques for neonatal aneurismal malformations of the vein of Galen. Pediatrics 1993;91:583-586

5. Fong LV, Lee SH, Salmon AP. Diagnosis of cerebral arteriovenous malformations by colour Doppler examination. Eur Heart J 1992; 111113:415-417.

6. Ford EG, Stanley, Tolo V, et al. Peripheral congenital arteriovenous fistulae: Observe, operate, or obdurate? J Pediatr Surg 1992;27:714-719

7. Lylyk P, Vineula F, Dion J E et al. Therapeutic alternatives for vein of Galen vascular malformations. Journal of Neurosurgery 1993; 78: 438-445.

\section{AUTHORS:}

1. Dinakara Prithviraj

2. Suresh A.

3. Anna Mariam Paul

\section{PARTICULARS OF CONTRIBUTORS:}

1. Associate Professor, Department of Paediatrics, VIMS \& RC.

2. Assistant Professor, Department of Radiology, VIMS \& RC.

3. Post Graduate, Department of Paediatrics, VIMS \& RC.
NAME ADDRESS EMAIL ID OF THE CORRESPONDING AUTHOR:

Dr. Dinakara Prithviraj,

Vydehi Institute of Medical Sciences and Research Centre, \#82, EPIP Area, White Field, Bangalore - 560066.

E-mail: drdinakar.nishanth@gmail.com

Date of Submission: 27/03/2014. Date of Peer Review: 28/03/2014. Date of Acceptance: 04/04/2014. Date of Publishing: 09/04/2014. 